\section{Perspective Epidemiology}

Check for updates

\title{
How far can African swine fever spread?
}

\author{
Jung-Hyang Sur $\mathbb{B}^{*}$
}

Department of Veterinary Pathology, College of Veterinary Medicine, Konkuk University, Seoul 02059, Korea

\begin{abstract}
African Swine Fever (ASF) is a highly contagious and deadly viral disease affecting both domestic pig and wild boar populations. Once introduced, it is a terrible disease that can devastate the swine industry in many countries. ASF has spread most recently beyond China to Southeast Asia, and parts of the Korean Peninsula. The majority of Asian countries consume pork as the primary source of meat compared to all other meat products. Particular emphasis is on the spread of ASF within North Korea and on future perspectives including protective guidelines. Thus far, the Korean peninsula has endured an extensive history of diseases, most notably from foot and mouth disease. For this reason, the Korean swine industries are familiar with the detrimental impacts of such a disease. On the other hand, exposure to a disease like ASF will decimate the swine industry even further. Therefore, it is crucial to bring urgent awareness to the spread of ASF.
\end{abstract}

Accepted: Jun 24,2019

*Corresponding author:

Jung-Hyang Sur

Department of Veterinary Pathology, College

of Veterinary Medicine, Konkuk University, 120

Neungdong-ro, Gwangjin-gu, Seoul 02059,

Korea.

E-mail: jsur@konkuk.ac.kr

(c) 2019 The Korean Society of Veterinary

Science

This is an Open Access article distributed under the terms of the Creative Commons Attribution Non-Commercial License (https:// creativecommons.org/licenses/by-nc/4.0) which permits unrestricted non-commercial use, distribution, and reproduction in any medium, provided the original work is properly cited.

ORCID iDs

Jung-Hyang Sur (D)

https://orcid.org/0000-0003-4496-7582

Conflict of Interest

The author declares no conflicts of interest.

Keywords: African swine fever; epidemiology

Only a few years ago, African swine fever (ASF) was a forgotten disease. Leading researchers believed it was merely a trivial concern for another country to solve. However, in recent years, ASF has entered the spotlight to pose as one of the most threatening epidemics in the swine industry, worldwide.

ASF is a highly contagious hemorrhagic disease found in domestic and wild pigs. The viral endemic originated in southern equatorial regions of Africa in warthogs and bush pigs, but, intriguingly, ASF viral infections in these African wild pigs remain asymptomatic. The ASF virus (ASFV) also infects soft ticks of the genus Ornithodoros, which act as natural reservoirs. ASF is typically characterized by high fever, inactivity, hemorrhages in the skin and internal organs, and death within a range of 2-10 days. Mortality rates may be as high as 100\% [1]. There is no vaccine or treatment for the ASFV. Fortunately, ASF cannot be transmitted to humans through direct contact with a pig. ASF is a disease of pigs only and therefore is not a threat to other livestock.

Briefly, the epidemiology of ASF began with the first recorded outbreak occurring in the Montgomery region of Kenya, Africa in 1921. Then, ASF crossed the continent to Europe and 
the virus was recorded on a pig farm near Lisbon, Portugal in 1957, causing $100 \%$ mortality. Since then, the disease has spread in western Europe, with records from Spain and Italy. In 2007, ASF spread to countries in the Caucasus region (Armenia, Azerbaijan, and Georgia) and to Russia. In particular, the outbreak in the Caucasus region has been reported to be the result of the illegal importation of ASF-contaminated meat from East Africa; subsequently, it was reported that the Russian outbreak in Caucasus border region, was due to wild boar movement [1,2]. Since the first reported outbreak in 2007, Russia and the countries in the Caucasus region have concluded that, since 2011, the outbreak of ASF has not been controlled since 2011.

The first outbreak of ASF in China occurred in Shenyang, a northeastern city of Liaoning province China in 2018. As of May 17, 2019, a total of 134 ASF outbreaks have been reported in China, including an outbreak in Hong Kong [3,4]. ASF continues to spread through Southeast Asia at a rapid pace. In fact, ASF seems to have already occupied most areas of China, Mongolia, Vietnam, and Cambodia and the most recently North Korea as well. If we back-track the advance of this epidemic, we observe that the disease has spread through the continent of Africa, and the continent of Europe, and finally through China to Southeast Asia, at an alarming rate. In light of this aggressive epidemic, the continent of Africa, a land distant from Korea by an entire ocean or continent, now feels closer to us than before. Current research predicts that ASF will continue to spread throughout Southeast Asia and China. Ultimately, this means that South Korea, as well as Japan, can no longer be considered an ASF safe zone.

In recent years, a report on the outbreak of ASF in China indicated that the disease might have been introduced from Eastern Caucasus countries via smuggled pork (illegal transactions) or through the disposal of waste foods (pork meat products) [3]. In connection with this estimate, whole-genome sequence analysis of ASFV within the Asian region, including China and Vietnam, can be used to identify possible origins of the virus and differentiate them from closely related strains. According to recent European whole-genome sequence analysis, which compares representative Eastern European ASFV Georgia 2007/1 with ASFV-China 2018/1 and Vietnam (VNUA/HY-ASF-1), sequences revealed an overall sequence identity of $99.98 \%$ at the nucleotide level [5]. Also, it was speculated that the disease had already become endemic in wild boar populations in several counties and was able to expand its range to include Eastern Europe and China, causing serious concerns. At this point, agencies within the Korean Peninsula must learn from the experiences of China and other neighboring countries, in order to prevent an outbreak in Korea.

In order to avoid repeating history and the further spread of ASF, a top priority is ASF prevention. Preventative measures can include avoiding the feeding of swill to pigs. Swill can contain serious exotic diseases that could devastate swine industries. For example, the ASFV can remain infective for 15 weeks in chilled meat (longer if frozen), and for 3 to 6 months in pork products such as cured hams and sausages that have not been cooked or smoked at a sufficiently high temperature. These characteristics have very crucial implications for the spread of ASF. Until now, most ASF outbreaks in Europe and Asia have been the result of illegal transactions involving infected pigs, pork products, and the distribution of swill-type feed. Based on this historical information, illegal importing and exporting of pork has been the dominant factor causing the ASF spread across countries (Table 1) [1,3].

Additionally, it is difficult to determine whether the transmission of ASF from wild boars to domestic pigs in the Korean Peninsula poses a general concern. This general concern is 
Table 1. Chronology of previous ASF outbreaks

\begin{tabular}{lll}
\hline Year & \multicolumn{1}{c}{ ASF outbreak country } & \\
\hline 1921 & Kenya* $^{*}$ & Unknown \\
1957 & Portugal & Pork or pig product from Africa \\
1960 & Spain & Pork or pig product \\
1971 & Cuba & Raw pork waste or pork product via an airplane from Portugal or Spain \\
1985 & Belgium & Pork or pig product \\
2007 & Georgia (Caucasus region) & Raw pork waste at an airport \\
2007 & Russia & Wild boar \\
2015 & Poland, Estonia & Wild boar \\
2018 & China & Pork or pig product \\
2019 & Mongolia & Pork or pig product \\
2019 & Vietnam & Pork or pig product \\
2019 & North Korea & Illegal importation of Pork or pig product \\
\end{tabular}

ASF, African swine fever.

*First recorded outbreak.

†Mongolia; Unpublished data. https://www.bloomberg.com/news/articles/2019-01-16/hogs-on-alert-in-asia-asafrican-swine-fever-spreads-to-mongolia.

‡North Korea; Unpublished data. https://www.rfa.org/korean/in_focus/ne-je-06052019171955.html.

associated with the geographical terrain of the Korean Peninsula and the presence of the demilitarized zone (DMZ) that serves as a physical buffer (Fig. 1). In order for ASF to reach South Korea, infected wild boars must cross 2 large channels in North Korea, as well as the DMZ. However, despite the geographical circumstances, we should always keep in mind that wild boars can spread ASF disease.

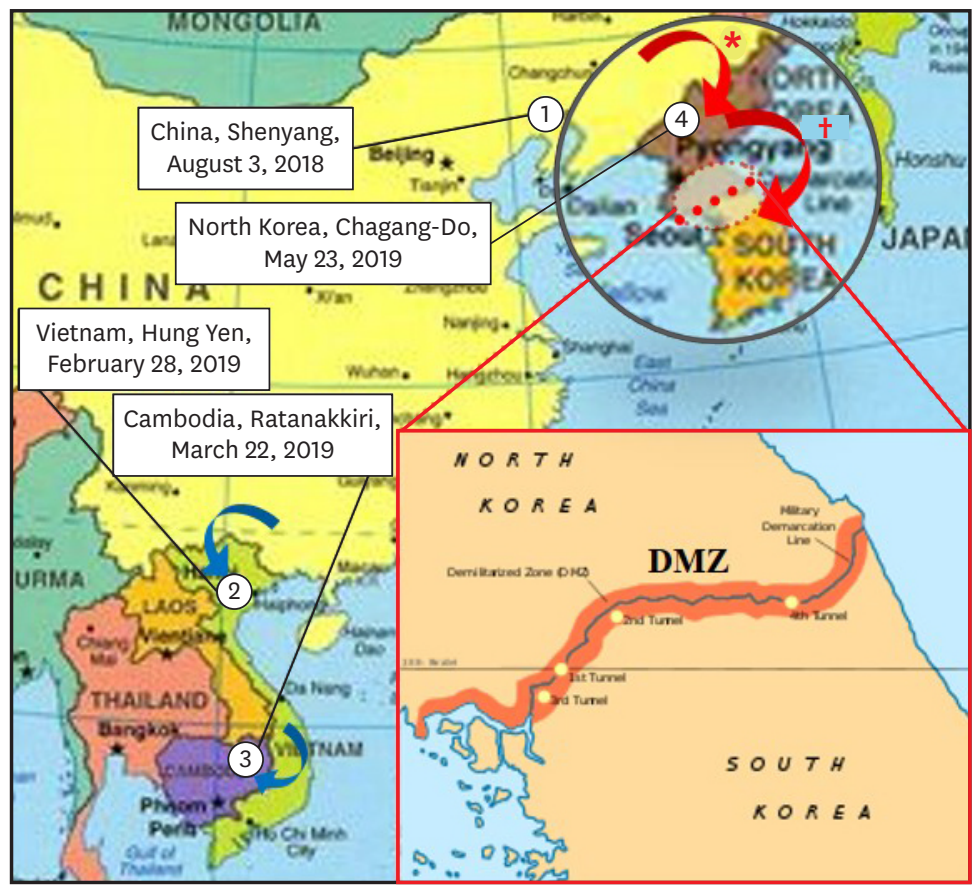

Fig. 1. African swine fever outbreak locations in Asia. The incidents are organized by year (China, Mongolia, Vietnam, Cambodia, North Korea). Arrows (curved red) in black circle indicate virtual wild boar migration path. Red dots are presented DMZ.

DMZ, demilitarized zone.

*North Korea is first expected path.

${ }^{+} \mathrm{DMZ}$ is second expected path.

Inset: details of DMZ, 250 kilometers (160 miles) long, and about 4 kilometers (2.5 miles) wide (source: Wikipedia, the free encyclopedia). 
What is being done to prevent or control this disease? Without a doubt, it would be best if an ASF vaccine was developed. However, despite ongoing international contributions to ASF research, we are still far away from creating a vaccine. So, what measures being taken for the development of the ASF vaccine? Several European and US groups have implemented new and diverse vaccine studies including the development of classic inactivated vaccines, live-attenuated vaccines, subunit vaccines, and so on, but at present, there is no effective vaccine against the ASFV on the market. So, what are the main causes of the failure to develop an ASF vaccine?

There are 2 critical factors that hinder the development of commercially available vaccine strategies against the ASFV. Firstly, the high complexity, immunity, and extensive nature of the DNA of the ASFV have complicated the creation of a vaccine. The ASFV is a large, double-stranded DNA virus that encodes more than 170 different proteins. The ASFV particle contains at least 50 proteins arranged in multiple layers. Secondly, the notable diversity among ASF strains means the virus encodes multiple virulence factors allowing its replication in porcine macrophages and concomitant evasion of the host's immune response, therefore hindering the development of efficient antiviral strategies $[2,6]$. To overcome such adversities, international joint efforts on vaccine development should be undertaken with the aim of obtaining a safe and efficient vaccine against ASF. Indifference to this pressing matter leads to the false perception that ASF vaccine development is an impossible task to pursue. As Henry Ford once said, "Failure is simply the opportunity to begin again, this time more intelligently."

Obviously, we already know how ASF can be effectively prevented. We can utilize the case studies from other countries as a basis for thorough implementation of preventative measures. The Korean government, mass media, and various associations are in charge of increasing public awareness and creating a sense of urgency about such topics as developing a strategy for disease prevention. Above all, active and strong cooperation is needed at the government level. Of course, the active cooperation of both livestock workers and ordinary citizens must also be encouraged. In summary, the key preventative measures are as follows:

- Countries with no ASF outbreak, including South Korea, must recognize how the disease can potentially infiltrate into non-ASF countries. Even though the likelihood of infection may be reduced by the presence of geographical barriers (such as large rivers), all countries should thoroughly analyze the potential routes of infiltration.

- Swill feeding must be strongly regulated by invoking stringent government measures.

- After visiting any country with an ASF outbreak, visitors must be kept in quarantine for 5 days. Moreover, the concealed smuggling of meat products in passengers' baggage represents a more significant risk to the introduction of animal diseases than that posed by the disposal of catering waste from international flights. Recently, The Korean government has significantly strengthened the law associated with smuggling. Failure to declare food products may result in up to 10,000,000 WON (App. 870 USD) in fines and penalties.

- Fences must be installed to block intruders such as roaming wild boar.

- Some countries with economically important agriculture sectors such as Australia, Canada, EU countries, New Zealand, and the USA have imposed strict controls on food waste from international flights, ships, and other international conveyances based on animal health concerns (https:/www.iata.org/whatwedo/environment/Documents/ summary-icw-case-for-smarter-regulation.pdf). Such controls require all international garbage to be disposed of appropriately and under the appropriate government enforcement system. Thus, internationally transported garbage must be moved under seal to an approved incineration facility. 
ASF is one of the deadliest viral diseases affecting swine herds and once a herd is infected, the effects cannot be reversed. Asia's characteristically high pork consumption rate makes it highly vulnerable to market fluctuations in the pork industry. Moreover, China is home to the world's largest swine herd. If ASF affects China's dominance in the production of pork, it could lead to a scarcity of pork and market upheaval. As crucial as disease prevention may be, so is the price stability in the eyes of the common man; therefore, stopping the spread of ASF is dually important.

\section{REFERENCES}

1. Sánchez-Vizcaíno JM, Mur L, Gomez-Villamandos JC, Carrasco L. An update on the epidemiology and pathology of African swine fever. J Comp Pathol 2015;152:9-21. PUBMED | CROSSREF

2. Sánchez EG, Pérez-Núñez D, Revilla Y. Development of vaccines against African swine fever virus. Virus Res 2019;265:150-155. PUBMED | CROSSREF

3. Wang T, Sun Y, Qiu HJ. African swine fever: an unprecedented disaster and challenge to China. Infect Dis Poverty 2018;7:111. PUBMED | CROSSREF

4. African Swine Fever (ASF). Report $\mathrm{N}^{\circ}$ 18: May 10-23, 2019 [Internet]. World Organisation for Animal Health; c2019 [updated 2019 May 10; cited 2019 May 23]. Available from: http://www.oie.int/fileadmin/ Home/eng/Animal_Health_in_the_World/docs/pdf/Disease_cards/ASF/Report_18_Current_situation_of_ ASF_mic.pdf.

5. Forth JH, Tignon M, Cay AB, Forth LF, Höper D, Blome S, Beer M. Comparative analysis of whole-genome sequence of African swine fever virus Belgium 2018/1. Emerg Infect Dis 2019;25:1249-1252. PUBMED | CROSSREF

6. Rock DL. Challenges for African swine fever vaccine development-"... perhaps the end of the beginning.". Vet Microbiol 2017;206:52-58.

PUBMED | CROSSREF 